\title{
Editorial \\ How deep is too deep in cardiac resuscitation
}

\author{
Volume 2 Issue 2 - 2015
}

\section{Editorial}

Every 5years the American Heart Association meets to update new CPR and ACLS guidelines based upon the data collected from the previous 5years (the newest guidelines were released 2010 and the next update will be released sometime in early 2016). Although the American Heart Association's 2010 CPR guidelines recommend compressing the chest in adults to a depth of at least 2 inches $(5 \mathrm{~cm})$ and in infants and children to a depth of least one third the anteriorposterior (AP) diameter of the chest or about $1 \frac{1}{2}$ inches $(4 \mathrm{~cm})$ in infants and about 2 inches $(5 \mathrm{~cm})$ in children, most people recognize that if the chest is significantly compressed more than that collateral damage to other internal organs and can decrease survival possibly from other contributing causes. Keep in mind that preserved integrity of the chest allows for the recoil. So what is the ideal compression depth and frequency for compressions? Should it be different in men versus women? Does going against the inspiration by a bag mask have any effect during the resuscitation? First, it needs to be recognized that survival depends on time to initiation of CPR and the quality of CPR as they dictate viability and recovery. Survival for majority of out of hospital resuscitation in such circumstances becomes dreadfully poor after the first few minutes of cardiac arrest. Second it takes a lot of energy, somewhere around 60pounds of pressure, to thrust the chest on average well-built adult male person to a depth of at least 2 inches $(>5 \mathrm{~cm})$. The average out of shape person would not be able to sustain that workout energy for a prolonged period of time. So initiation of emergency medical service is very important. In most circumstances, the main consideration is not organ damage from too deep a chest compression but insufficient depth to obtain good circulation. In chest compression only, insufflation/air exchange would be another consideration that would be compromised. A compression rate of 100 to 120 per minute is reported to be the sweet spot for optimal resuscitation and goal of survival. But like any bell shaped curve, some responders are giving chest compressions too fast, with about a third above 120 compressions per minute, and others 20 percent giving them above 140per minute.

From the work performed at the University of Arizona, Cardiocerebral Resuscitation advocates compression only CPR for primary cardiac arrests (not a drowning victim). Call for help and start chest compressions. The reason for this change was that the requirement of mouth-to-mouth ventilation as the initial step of bystander resuscitation prevented many, including professionals, from initiating bystander CPR. In addition, the chest compression itself in studies shown to provide sufficient insufflation for exchange of air and ventilation. The hemoglobin oxygen delivery curve plays a role.

It is very important to cut excessive delays or interruptions of chest compressions occurring from interrupted CPR and subsequent deprivation of circulation. During the electrical phase of the cardiac arrest, time to attachment of the pads and eventually shock delivery

\author{
Brian Boudi F \\ Phoenix VA Health Care System, University of Arizona, USA
}

Correspondence: Brian Boudi F, Phoenix VA Health Care System, University of Arizona, College of Medicine Regional Faculty, American Heart Association, USA,

Email bII534@aol.com

Received: February 16, 2015 | Published: March 06, 2015

should seek to keep the circulation flow uninterrupted to sustain survival by priming the system with ATP so the electrical shock have a chance of converting underlying ventricular fibrillation into a sustainable rhythm. In addition, chest compressions should be initiated immediately post electrical shock for at least 2 minutes in order to perfuse the heart and increase the likelihood that it will be able to generate adequate arterial pressures. So going back to how deep is too deep, so long as the quality compressions are well positioned directly over the chest in a vertical direction over center of the chest, occurring to more than 100per minute with allowance of full recoil and depth of at least 2inches, and until clinical studies better define how deep would be considered too deep, It is better to go deep than not deep enough. Bones and organs can heal, but a dead heart will not. In terms of post resuscitative reassessment/review, if the chest recoil is very significantly compromised (excluding individuals with extreme age and osteoporosis), then it would be secondary indication that the compressions may have been carried out too deep. Press hard and fast but allow for full recoil.

\section{Acknowledgments}

None.

\section{Conflicts of interest}

Author declares there atre no conflicts of interest.

\section{Funding}

None. 